\title{
Views of institutional leaders on maintaining humanism in today's practice
}

\section{Running Head: Views of institutional leaders}

MaryAnn C. Gilligan, M.D., M.P.H' ${ }^{1}$

Lars G. Osterberg, M.D., M.P.H. ${ }^{2}$

Elizabeth A. Rider, M.S.W., M.D. ${ }^{3}$

Arthur R. Derse, M.D., J.D. ${ }^{4}$

Amy B. Weil, M.D. ${ }^{5}$

Debra K. Litzelman, M.D. ${ }^{6}$

Dana W. Dunne, M.D. ${ }^{7}$

Janet P. Hafler, Ed.D. ${ }^{8}$

Margaret Plews-Ogan, M.D. ${ }^{9}$

Richard M. Frankel, Ph.D. ${ }^{10}$

William T. Branch, Jr., M.D. ${ }^{11}$

${ }^{1}$ Division of General Internal Medicine, Department of Medicine, Medical College of Wisconsin, Milwaukee, WI., USA. Mgilliga@ mcw.edu. ${ }^{2}$ Department of Medicine, Stanford University School of Medicine, Palo Alto, CA., USA. Larso@stanford.edu. ${ }^{3}$ Department of Pediatrics, Harvard Medical School; and Division of General Pediatrics, Department of Pediatrics, Boston Children's Hospital, Boston, MA., USA. Elizabeth.Rider @ hms.harvard.edu. ${ }^{4}$ Center for Bioethics and Medical Humanities, Institute for Health and Equity and Department of Emergency Medicine, Medical College of Wisconsin, Milwaukee, WI., USA. Aderse@mcw.edu. ${ }^{5}$ Department of Medicine, University of North Carolina School of Medicine, Chapel Hill, NC., USA. Amy_Weil@med.unc.edu. ${ }^{6}$ Indiana University School of Medicine, Indianapolis, IN., USA. Dklitzel@iu.edu. Yale University School of Medicine, ${ }^{7}$ Department of Internal Medicine, New Haven, CT., USA. Dana.Dunne@ Yale.edu. ${ }^{8}$ Department of Pediatrics, Yale University School of Medicine, New Haven, CT., USA. Janet.Hafler@ Yale.edu. ${ }^{9}$ Division of General, Geriatric, Palliative and Hospital Medicine, Department of Medicine, University of Virginia School of Medicine, Charlottesville, VA., USA. Mp5k@hscmail.mcc.virginia.edu. ${ }^{10}$ Department of Medicine, Indiana University School of Medicine, Indianapolis, IN and Education Institute, Cleveland Clinic, Cleveland, OH., USA. Rfrankel@iu.edu. ${ }^{11}$ Division of General Medicine and Geriatrics, Department of Medicine, Emory University School of Medicine, Atlanta, GA., USA. Wbranch@emory.edu. 
Support:

Arnold P Gold Foundation [grant number FI-14-008]

IRB Approval or Exemption: Yes

Corresponding Author:

William T Branch, Jr, MD

Carter Smith, Sr. Professor of Medicine

Department of Medicine

Emory University School of Medicine

49 Jesse Hill Jr. Drive

Atlanta, GA 30303

Phone: 4047781610

Fax: 4047781601

Email: Wbranch@emory.edu

Word count: 3678

Abstract: 200

References: 44

Highlights

- US healthcare leaders sympathize with clinician stress and frustration.

- Leaders support programs that encourage humanistic skills, values and attitudes.

- Faculty development activities are not coordinated for maximal impact.

- Leaders have not considered organizational humanistic practice changes.

- Organizational changes may be most effective to preserve medical humanism.

\begin{abstract}
OBJECTIVE: To explore leadership perspectives on how to maintain high quality efficient care that is also person-centered and humanistic.

METHODS: The authors interviewed and collected narrative transcripts from a convenience sample of 32 institutional healthcare leaders at seven U.S. medical schools. The institutional leaders were asked to identify factors that either promoted or inhibited humanistic practice. A subset of authors used the constant comparative method to perform qualitative analysis of the
\end{abstract}


interview transcripts. They reached thematic saturation by consensus on the major themes and illustrative examples after six conference calls.

RESULTS: Institutional healthcare leaders supported vision statements, policies, organized educational and faculty development programs, role modeling including their own, and recognition of informal acts of kindness to promote and maintain humanistic patient-care. These measures were described individually rather than as components of a coordinated plan. Few healthcare leaders mentioned plans for organizational or systems changes to promote humanistic clinician-patient relationships.

CONCLUSIONS: Institutional leaders assisted clinicians in dealing with stressful practices in beneficial ways but fell short of envisaging systems approaches that improve practice organization to encourage humanistic care.

PRACTICE IMPLICATIONS: To preserve humanistic care requires system changes as well as programs to enhance skills and foster humanistic values and attitudes.

Key words: humanism, organizational culture, faculty development, burnout, leadership, values, compassionate healthcare

\section{INTRODUCTION}

1.1 From its beginning, medicine as a profession has emphasized humanistic values such as benevolence and compassion. [1] Scribonius, a physician in $47 \mathrm{CE}$, defined the profession as "a commitment to compassion....in the relief of suffering." [2] In current times, medical humanism refers to "deep-seated convictions of physicians", such as to be caring, compassionate, and respectful toward patients and colleagues. [3-9] Cohen states that a physician's humanistic convictions animate professionalism, which incorporates corresponding behaviors. [3] Professionalism and humanism are considered essential to the practice of medicine. [5-9] 
In the United States, emphasis on clinical productivity, efficiency, performance metrics, and regulations has created barriers to the humanistic practice of medicine that has contributed to high levels of frustration, stress, and burnout among physicians and other clinicians. [10-12] The challenge to those leading healthcare organizations, then, is how to respond to the conflicting goals and values of humanistic practice and economic efficiency. What steps have the leaders taken to ensure that the humanistic tradition is preserved, while also achieving efficient, high quality care? Both academic leaders and healthcare administrators share this responsibility and should articulate a vision that bridges the current divide between humanistic care and economic imperatives. [13]

In our previous work, we interviewed physicians who had completed a faculty development course in humanistic medicine at 7 medical schools across the U.S. about factors within their organizations that either promoted or inhibited humanistic practice. [14,15] Here, we report on our interviews with healthcare leaders at those same medical schools to learn from their perspectives, the actions they have taken, and their views of how to maintain high quality, efficient, person-centered, humanistic care.

\section{METHODS}

2.1 Design: A qualitative research design was implemented to explore leadership perspectives on how to maintain high quality efficient care that is also person-centered and humanistic

Sample: Participants were invited to enroll in the study by the local facilitators at each school, who are also co-authors of this paper. We asked facilitators to invite healthcare leaders with 
influential roles who were thought likely to participate in a semi-structured interview. Institutional healthcare leaders included in this study were a convenience sample of 32 participants who met these criteria and consented to be interviewed. The study was exempted or approved by the Institutional Review Board at each institution.

Participants were $38 \%$ female; more than half were between 45 and 64 years of age. The majority were physicians (81\%). Administrative titles included Dean, Associate or Assistant Dean (8), Department Chair or Vice Chair (7), Division Director (4), CEO, Director, or Chief Medical Officer (4), Nursing Leader (2) and miscellaneous institutional healthcare leadership roles. Based on their titles, 46 per cent held administrative titles indicating responsibilities for both clinical and educational programs (e.g., Department Chair), 16 per cent were administrators responsible for the practices (e.g., CEO), and 38 per cent were leaders of medical education (e.g., Education Dean). All are henceforth referred to as "leaders."

Data Collection: Interviews were conducted in-person or via email, after which leaders responded to an online questionnaire requiring narrative answers. Of the 32 interviews, 11 were from school \#1, 6 from \#2, 5 from \#3, 3 from \#4, 3 from \#5, 3 from \#6, and 1 from \#7. We provided participants with a working definition of medical humanism taken from the Arnold P. Gold Foundation [7] on which to base their answers. Medical humanism was defined in the interview as "characterized by respectful and compassionate relationships among physicians, their patients, and other members of the healthcare team that flourishes within a humanistic culture." [7] Leaders were asked to provide written reflective responses of one or more paragraphs addressing two prompts: (a) What factors and/or programs in your organization encourage practicing and teaching humanistically? and (b) What factors in your organization 
pose barriers to creating a humanistic practice and/or environment? What proposed solutions are you considering? Responses averaged 93.9 words (95\% confidence intervals 75.0 to 113.0 words, std. dev. 75.9 words, median 78.5 words, range 2-315 words).

2.2 Data Analysis: Transcripts were analyzed using the constant comparative method. [16-19] Five co-authors (WTB, MAG, EAR, LGO, ARD) participated in the analysis, which included attendance on six conference calls each lasting 90-120 minutes. The written responses from participant interviews were circulated before each call for review by these five investigators. Typically, during each call, a subset of 5 or 6 written responses was read aloud one-by-one, discussed, and themes identified. On each subsequent call, themes that had been previously identified were reviewed and revised if necessary to reflect the discussion of the new subset. Illustrative phrases related to the themes were recorded. By the sixth call, all participantresponses had been analyzed and no additional or more meaningful revisions of the themes were identified leading investigators to conclude that they had reached thematic saturation.

\section{RESULTS}

Our analysis led us to group the factors that encouraged humanism and that posed barriers under three broad themes. This approach allowed us to compare and contrast the leaders' views of factors and subthemes (in bold) related to maintaining humanistic practice (Table 1).

\subsection{Theme 1: Challenges and Responses of Leadership}

3.1.1 The leaders uniformly indicated that they cared about maintaining humanistic practice. They described and often acknowledged the overwhelming amount of work confronting clinicians and the resultant stresses: 
"In our very busy hospital and clinic environments, there are so many things our providers and staff need to attend to that they are often overwhelmed and simply feel the need to move on to the next patient."

\subsubsection{Leaders were aware of the added challenges of providing humanistic care to patients}

following the introduction of the electronic medical record (EMR), due to both the physical intrusion of computers into clinical encounters and the added administrative burden that they placed on clinicians:

"There is a certain degree of burnout that is endogenous to clinical work, and it has only gotten worse with the forced implementation of the electronic medical record that oftentimes force us to just keep clicking and spend far less time in direct patient contact."

\subsubsection{Financial pressures were acknowledged as a challenge to humanistic care:}

"I think the greatest barriers are time, [and] money...There are increasing pressures on faculty and residents to work harder and take on more work that stresses their ability to practice and teach in a humanistic manner."

\subsubsection{Leaders recognized the critical importance of good faculty role models:}

"I think role modeling may be the single most effective practice to encourage humanism, in addition to efforts to recognize and EXPLICITLY teach and comment and evaluate upon elements of humanistic practice."

\subsubsection{One leader, who was also a clinician, expressed his dissatisfaction with the tension}

between modeling humanistic practice and being responsive to productivity pressures using language that suggested resignation to a loss of idealism replaced by growing cynicism:

So the big issue is: We are encouraged and most directly rewarded to do the things that are the least humanistic. We might perfect the ability to bill and collect while, at the same time, lose all ability to care empathically for patients. And our colleagues, our students, and our residents watch us while we do this.

\subsubsection{Leaders also voiced concern about the increasing fragmentation in the structure of}

healthcare delivery that inhibited continuity in patient-relationships - from the era in which 
primary care attending physicians provided care to their own patients in the hospital to a hospitalist model, and from unlimited work hours to duty hour restrictions for trainees.

\subsection{Theme 2: Policies, Programs and Practices that Promote Humanistic Care}

3.2.1 Official "top-down" policies were often named by leaders as promoting humanistic care, including mandated tracking of patient satisfaction and mission and vision statements of the organization.

A humanistic approach to teaching and practicing medicine is identified in the institutional and departmental values communicated to the faculty, staff, residents and students. I believe that in order to encourage a humanistic approach the institution must be able to define it, identify it, and assess it.

One individual articulated the responsibility of leaders to enforce these policies along with a vision describing "the essential nature of institutional humanism":

A clear leadership/operational structure - that promotes an environment that facilitates trust - is essential to humanism/professionalism. Without clear responsibility and accountability - a humanistic environment can be gradually eroded despite the best intentions of individual providers. The essential nature of institutional humanism is as (if not more) important than individual humanism/professionalism.

Some policies created by leaders promoted humanistic behavior through recognition and rewards:

The school recognizes teamwork like never before in all three missions--teamwork in patient care to ensure the best experience a patient can have, teamwork in science to ensure that basic discoveries translate into patient benefit, and teamwork in education that has changed our focus from teaching to learning.

\subsubsection{Another common response was to list organized educational and faculty development}

programs; for example, the Healer's Art course, bioethics curricular threads, offices of faculty affairs and diversity, women's faculty council seminars, and required yearly sessions on diversity, burnout and self-care [20-25]. These programs varied in the degree to which the 
leaders were involved in planning, guiding, and implementing them. The programs taught caring attitudes, diversity, mindfulness, and communication and related humanistic skills and attitudes, using mostly discussion, small-group and other active learning methods. [20-25] For the most part, the educational and faculty development programs were not created to be part of a coordinated plan to address the growing barriers to humanistic practice. Most were "bottom-up" programs reflecting the interests of individual faculty members, although some of the individual programs (e.g., “...we implemented the National Coalition Building Institute program to raise awareness of the need to treat all individuals with dignity and respect.") reflected a school policy initiative.

3.2.3 In a few schools, faculty and students created special programs because they perceived the need, often in response to an event that was an informal example of humanism or kindness: ...one of our 3rd year medical students died in a car accident. Her classmates were devastated. Immediately after her death, they held a candlelight vigil and about a month later developed a beautiful memorial service, which her family attended. But they were obviously still struggling with their thoughts and feelings. In response, we developed a two-hour evening session for the students that provided an opportunity to talk with each other and with faculty.

At other times, participation in events outside of the formal curriculum, such as a memorial service organized by a deceased patient's family members, had an impact on faculty members and learners who attended. An example was this memorial service:

After a peaceful death for the patient, I was able to attend and participate in a wonderful memorial service at a rural African American Church, which was a moving memory and tribute to this woman who turned out to be a pioneer in civil rights in local history. The nephew was recognized for his wonderful care of the patient and her 94 year old sister for many years and the community (and several attendees from the care team) were energized by their involvement in service. Warm feelings all around! 
These informal responses to events were not part of a coordinated response but reflected the desire of leaders and faculty members to respond appropriately. One leader acknowledged the lack of coordination of the programs at her institution, at the same time expressing the hope that the programs would still have the desired impact:

Multiple programs run throughout the curriculum, although they are not coordinated...The overall effect, though, is to create a culture where these opportunities are available and hopefully a critical mass of people at all levels that see that Humanism is important.

\subsection{Theme 3: Organizational Factors and Changes}

3.3.1 Efforts to influence the organization and its culture included both top-down and bottomup approaches. Many leaders identified activities or training programs developed and implemented from the top-down. For example, one wrote:

"We provide tools, training and scripts to ensure all staff and physicians are asking standard questions and providing standard information to patients and families to ensure they are put at ease."

However, the same individual went on to describe how such top-down approaches can backfire:

"While I believe we have put in place a standard message emphasizing "patient first" and providing some support, training and tools, these same things can also create barriers to creating a humanistic environment. Our staff and physicians give us feedback that they often feel disconnected from the patient while they are focusing on the standard questions and scripts."

Setting clear expectations of acceptable behavior was one of the top-down approaches used by

leadership:

"By making behavioral expectations explicit and enforcing policies governing the way we treat each other, the culture is changing in a positive way."

If expectations were clear, then leaders felt they had the power to address the "sweeping under the carpet egregious behaviors of faculty too important to [ignore]," and "to summon the 
courage to actually remove people from the environment if they consistently interfere with the humanistic mission of the institution."

One leader expressed the need for a bottom-up approach reflecting the kind of physicians he would like to have in his organization:

"It's about developing a caring attitude towards other human beings that you walk the planet with..."

3.3.2 Other than establishing policies and setting standards, only a few leaders described efforts by themselves or their institutions to alter organizational structure through systems change that would facilitate humanistic practice and potentially diminish burnout among clinicians. In one instance, a leader articulated a plan for restructuring the medical practice to promote humanism:

"Financial incentives distort humanistic behaviors - we are trying to move away from RVU based rewards that can lead to destructive behaviors."

\section{DISCUSSION AND CONCLUSIONS}

4.1.1 Leaders were aware of organizational factors that inhibited humanistic care and seemed genuinely concerned about physician stress, dissatisfaction and burnout. When asked how they and their institutions would maintain humanistic care in the face of these factors, they cited institutional policies and vision statements, role modeling, organized educational and faculty development programs, informal teaching, and recognition of acts of kindness. With few exceptions, the organized programs reflected individual interests or single policies rather than coordinated plans to preserve or promote humanistic practice. These educational programs were available but not required for faculty members. Many leaders also cited a tradition of humanistic care at their institution, sometimes articulated in its mission statement, while others cited policies 
that evaluated patient satisfaction and provided feedback on patient-centered communication. Policies most often came from the top down, although some leaders acknowledged that top down policies could backfire if they created demands perceived as excessive by clinicians.

Role modeling was frequently mentioned as the primary method that encouraged humanism, which is consistent with a growing body of literature on this topic. [26-29]. Leaders sometimes specified being attentive to their own behavior or personal efforts to model humanism. This is important because the faculty physicians in our previous studies only gave credence to leaders who "talked the talk" if they also "walked the walk." [14-15]

Leaders commonly mentioned faculty development and educational programs that promoted humanism, but they only occasionally indicated personally initiating or actively participating in one of these programs. None of the leaders indicated that the educational programs were part of a comprehensive curriculum or coordinated strategy to promote medical humanism, although some individual programs were created specifically to promote humanistic practice, and one school had implemented a comprehensive school-wide humanistic program in the past. As further examples, some leaders pointed to individual practitioners, who set examples of going beyond usual expectations to assist or comfort a patient. These acts of kindness were sometimes memorialized by the practice community.

Although leaders indicated their support for traditions of humanism and lauded the aforementioned humanistic programs and acts of kindness, they mentioned no urgent need to alter practice-systems to make them more conducive to providing humanistic care. 
Our overall impression was that academic medical centers are engaged in a tug-of-war pitting leaders and professional staff, who wish to maintain humanism, against business expectations and outside forces, such as reimbursement schemes. Our results suggest that the tensions existing in practice environments might be partly counterbalanced by good role modeling, humanistic educational programs, and administrative policies that enhance and uphold humanistic traditions. However, surveys indicate burnout in 40 to 60 percent of physicians and other medical professionals [30-31], which is a threat to overall performance. [12, 32-33] Humanistic educational programs are thought to ameliorate burnout in part by enhancing meaning in practice, which bolsters resilience. [34-35] In addition, leaders are being called on to implement faculty development programs that promote mindful practice. [36] While these programs are helpful at the individual level, we agree with others who question whether they address the root causes of burnout at the organizational level. [37-38] In our study, leaders recognized that there were problems with the system of practice but did not articulate solutions which would ameliorate these problems, such as inadequate physician face-time with patients, administrative distractions, and excessive paperwork. Leaders did not mention collaborating with practicing front-line physicians and other stakeholders to re-organize systems to promote joyful, humanistic practice. We believe achieving this may require systems changes that provide a practice structure that better enables practitioners to build meaningful therapeutic patient-relationships.

We should ask why well-meaning, seasoned leaders, who were aware of issues in the practices, failed to apply the basic step of improving the systems they work in and are responsible for. [13] Perhaps they did not think that addressing systems change was part of the specific task of addressing barriers to humanism, but this seems unlikely because we explicitly asked, "What 
proposed solutions are you considering?" In addition, it is possible that leaders failed to appreciate the importance of going beyond educational programs to improve systems.

Other possible explanations for this disconnect between recognized systems barriers and systems-level solutions might be that the leaders considered the changes required to fix the system were stymied by outside forces beyond their level of management. This would imply a lack of agency to effect change within the organization, trapped in a box or "iron cage," as it were, as described by the sociologist Max Weber. [39] We found a similar lack of agency in our surveys of the clinical faculty physicians. [14,15]

The system itself is failing if, as one leader stated above, "We might perfect the ability to bill and collect while, at the same time, lose all ability to care empathically for patients." We can predict that clinical practice is in danger of remaining in crisis for some time to come until systems-level thinking takes hold, and leaders in health care organizations both recognize and exercise their agency over these systems-level problems.

The challenges in healthcare today are sometimes technical and sometimes adaptive. A technical challenge is one that can be solved mechanistically. Adaptive challenges require attention to emergent processes that cannot be predicted in advance and are a product of the interaction between individuals and the culture of the organization in which they occur. [40-41] Leaders commonly misidentify adaptive challenges as technical ones and incorrectly apply technical solutions. [41-43] However, adaptive challenges require a different approach involving changes in people's priorities, beliefs, and habits. We know that every system is perfectly designed to get the results it currently gets, and that change is hard because powerful forces are at work to 
maintain the status quo. Challenging the status quo in medicine is difficult but not impossible. It requires new ways of thinking and taking the risk of disrupting sociopolitical relationships. [32] However, until leaders are willing to rise to adaptive challenges and "dance on the edge of authority" the system will not improve. [40]

Fortunately, signs of bold leadership are beginning to emerge, although much more needs to be done. The Institute for Healthcare Improvement (IHI) Leadership Alliance, a collaboration of healthcare executives and their teams, have updated the "10 simple rules" to guide the redesign of healthcare delivery in the $21^{\text {st }}$ century, and they encourage leaders to be courageous and committed in finding and testing new ideas. [44-45] The IHI cites several examples of healthcare systems that are trying to cultivate wellbeing in the healthcare work force through pride and joy among other things. [45] In a study to address the increased clerical burden associated with the introduction of the electronic health record (EHR) and with inefficiency in the practice environment, Linzer, et al, conducted a promising randomized trial of interventions to improve work conditions (e.g., offloading nonessential tasks to non-physician staff, improving flow in and out of exam rooms) that consequently permitted increased patient-visit times. [46] Several studies have looked at the use of scribes to decrease the EHR documentation burden on physicians and found an increase in physician satisfaction without compromising patient satisfaction. [47-48] Some of the work that needs to be done (e.g., decreasing the current burden of documentation to meet billing and quality reporting requirements, pre-approval process for tests and procedures, increasing reimbursement for direct patient care relative to reimbursement for performing procedures) is perhaps beyond leaders' control, but would likely benefit from advocacy by institutional leaders. [49] 


\subsubsection{Limitations}

Our study is limited because we did not systematically enroll participants. Consequently, the results cannot be generalized. Nevertheless, we solicited influential local leaders at high level positions whom we would expect to be involved in improving care. Future studies, using systematic recruitment methods and nationally representative samples will determine how our findings fit into the larger picture of healthcare leadership in the United States today. Likewise, our findings at seven medical schools might not apply to all medical schools or other practice environments. To our knowledge, this is the first study to explore the healthcare leaders' perspectives on maintaining humanism in today's clinical environment. Qualitative research is a valuable and appropriate "point of departure" for investigating phenomena especially those that are new or under-researched.

\subsection{Conclusions}

Our study found well intentioned and engaged institutional healthcare leaders who cared about medicine's humanistic traditions. They understood the difficulties of practicing physicians and supported educational programs and policies designed to strengthen physician wellbeing and resilience. However, these same leaders were not sure how to prevent erosion of humanistic practice environments, or were generally not inclined to "rock the boat" by making wholesale changes to the practice organization at the time they were interviewed. We believe that bold vision and new practice structures will be necessary to solve, not simply mitigate, current impediments to physicians' humanistic care of their patients. The question of whether we, as a society and as a profession, can afford the structural changes that are needed to bridge the divide between economic priorities and humanistic care is perhaps better posed as, can we afford not to make the changes? 


\subsection{Practice Implications}

Institutional healthcare leaders should seek to maintain medicine's humanistic traditions of care by:

Supporting educational programs and institutional infrastructure and policies that promote humanistic skills, values and attitudes in their organizations

Improving the practice environment and systems of care in ways that maximize clinicians' ability to build and maintain humanistic therapeutic relationships with their patients and families.

Conflicts of Interest: None 


\section{REFERENCES}

[1] Pellegrino ED. Professionalism, profession and the virtues of the good physician. Mt Sinai J Med. 2002;69:378-384.

[2] Swick HM. Professionalism and humanism beyond the academic health center. Acad Med. 2007;82:1022-1028.

[3] Cohen JJ. Linking professionalism to humanism: what it means, why it matters. Acad Med. 2007;82:1029-1032.

[4] Chou CM, Kellom K, Shea JA. Attitudes and habits of highly humanistic physicians. Acad Med. 2014;89:1252-1258.

[5] American Board of Internal Medicine (ABIM). ABIM Committee on Evaluation of Clinical Competence and ABIM Clinical Competence and Communications Programs. Project Professionalism. Philadelphia: American Board of Internal Medicine Press. 1995 (seventh printing 2001).

[6] ABIM Foundation; ACP-ASIM Foundation; European Federation of Internal Medicine. Medical professionalism in the new millennium: A physician charter. Ann Intern Med. 2002; 136:243-246.

[7] Arnold P. Gold Foundation. What is humanism in medicine? Revised 2013. http://humanismin-medicine.org/index.php/aboutus/what_is_humanism_in_medicine. Accessed 10 April 2017.

[8] Rider EA, Kurtz S, Slade D, et al. The International Charter for Human Values in Healthcare: An interprofessional global collaboration to enhance values and communication in healthcare. Patient Educ Couns. 2014;96:273-280.

[9] Egener BE, Mason DJ, McDonald WJ, Okun S, Gaines ME, Fleming DA et al. The Charter on Professionalism for Health Care Organizations. Acad Med. 2017; 92:1091-1099. 
[10] Dugdale DC, Epstein R, Pantilat SZ. Time and the patient-physician relationship. J Gen Intern Med. 1999;14 (S1):S34-40.

[11] Sinsky C, Colligan L, Li L, Prgomet M, Reynolds S, Goeders L et al. Allocation of physician time in ambulatory practice: A time and motion study in 4 specialties. Ann Intern Med 2016;165:753-760.

[12] Dyrbye LN, Shanafelt TD, Sinsky CA, Cipriano PF, Bhatt J, Ommaya A, West CP, Meyers D. Burnout among health care professionals: A call to explore and address this under-recognized threat to safe, high-quality care. NAM Perspectives 2017. Discussion Paper, National Academy of Medicine, Washington DC.

[13] Schein EH. Organizational Culture and Leadership. $5^{\text {th }}$ ed. San Francisco, CA: John Wiley \& Sons; 2016.

[14] Branch WT Jr, Weil AB, Gilligan MAC, Litzelman DK, Hafler JP, Plews-Ogan M, Rider EA, Osterberg LG, Dunne D, Derse AR, Pittman JR, Frankel RM. How physicians draw satisfaction and overcome barriers in their practices: "It sustains me". Patient Educ Couns. 2017: $2320-2330$.

[15] Rider EA, Gilligan MAC, Osterberg LG, Litzelman DK, Plews-Ogan M, Weil AB, Dunne D, Hafler, JP, May NB, Derse AR, Frankel RM, Branch WT Jr. Healthcare at the Crossroads: The Need to Shape an Organizational Culture of Humanistic Teaching and Practice. J Gen Intern Med. 2018;33:1092-9.

[16] Glaser BG, Strauss AL. The constant comparative method in qualitative analysis. In: The Discovery of Grounded Theory: Strategies for Qualitative Research. Chicago, IL: Aldine Transaction; 1967. 
[17] Morse JM, Field PA. Qualitative Research Methods for Health Professionals. Thousand Oaks, CA: Sage; 1995.

[18] Stern PM, Kerry J. Grounded theory methodology. In: Morse JM, Stern PN, Corbin J,

Bowers B, Charmz K, Clarke A, eds. Developing Grounded Theory: The Second Generation.

Walnut Creek, CA: Left Coast Press; 2009.

[19] Miles MB, Huberman AM, Saldana J. Qualitative Data Analysis: A Methods Sourcebook. Thousand Oaks, CA: SAGE Publications Inc; 2013.

[20] Lown BA, Manning CF. The Schwartz Center Rounds: evaluation of an interdisciplinary approach to enhancing patient-centered communication, teamwork, and provider support. Acad Med. 2010;85:1073-1081.

[21] O'Donnell JF, Rabow MW, Remen RN. The Healer's Art: Awakening the heart of medicine. AACH Medical Encounter. 2007;21 (1):7-11.

[22] Rabow MW, Newman M, Remen RN. Teaching in relationship: the impact on faculty of teaching "the Healer's Art". Teach Learn Med. 2014;26:121-128.

[23] Kjeldmand D, Holmstrom I. Balint groups as a means to increase job satisfaction and prevent burnout among general practitioners. Ann Fam Med. 2008;6:138-145.

[24] Wald HS, Borkan JM, Taylor JS, Anthony D, Reis SP. Fostering and evaluating reflective capacity in medical education: Developing the REFLECT rubric for assessing reflective writing. Acad Med. 2012;87:41-50.

[25] Branch WT JR, Frankel RM, Hafler JP et al. A multi-institutional longitudinal faculty development program in humanism supports the professional development of faculty teachers. Acad Med. 2017;92:1680-1686. 
[26] Weissman PF, Branch WT, Gracey CF, Haidet P, Frankel RM. Role modeling humanistic behavior: learning bedside manner from the expert. Acad Med. 2006;81:661-7. [27] Birden H, Glass N, Wilson I, et al. Teaching professionalism in medical education: A Best Evidence Medical Education (BEME) systematic review. BEME Guide No. 25. Medical Teacher 2013;35:e1252-e1256.

[28] Passi V, Johnson S, Peile E, et al. Doctor role modelling in medical education: BEME Guide No. 27. Medical Teacher 2013;35:e1422-e1436.

[29] Yoon JD, Ham SA, Reddy ST, Curlin FA. Role models' influence on specialy choice for residency training: A national longitudinal study. J Grad Med Ed 2018;149-154.

[30] Shanafelt TD, Boone S, Tan et al. Burnout and satisfaction with work-life balance among US physicians relative to the general US population. Arch Intern Med. 2012;172:1377-85.

[31] Shanafelt TD, Hasan O, Dyrbye LN, et al. Changes in burnout and satisfaction with worklife balance in physicians and the general US working population between 2011 and 2014. Mayo Clinic Proc. 2015;90:1600-1613.

[32] Montgomery A, Panagopoulou E, Keho I, Valkanos E. Connecting organisational culture and quality of care in the hospital: is job burnout the missing link? Journal of Health Organization and Management 2011:108-123.

[33] Montgomery A, Todorova I, Baban A, Panagopoulou E. Improving quality and safety in the hospital: the link between organizational culture, burnout, and quality of care. Br J Health Psychol 2013 Sept;18:656-662.

[34] West CP, Dyrbye LN, Erwin PJ, Shanafelt TD. Interventions to prevent and reduce physician burnout: a systematic review and meta-analysis. The Lancet 2016 Nov 11;388:227281. 
[35] Schrijver I, Brady KJ, Trockel M. An exploration of key issues and potential solutions that impact physician wellbeing and professional fulfillment at an academic center. PeerJ. 2016 March 10;4:e1783.

[36] Shanafelt TD and Noseworthy JH. Executive leadership and physician well-being: Nine organizational strategies to promote engagement and reduce burnout. Mayo Clin Proc 2016: 118.

[37] West CP, Dyrbye LN, Rabatin JT, et al. Intervention to promote physician well being, job satisfaction nad professionalism: a randomized clinical trial. JAMA Intern Med. 2014; 174:527533.

[38] Sinsky CA, Willard-Grace R, Schutzbank AM, et al. In search of joy in practice: A report of 23 High-functioning primary care practices. Ann Fam Med. 2013; 11: 272-278.

[39] Goleman D, Boyatzis R, McKee A. Primary leadership: Learning to lead with emotional intelligence. Boston, MA, Harvard Business School Press, 2002.

[40] Heifetz RA, Grashou A, Linsky, M. The practice of adaptive leadership. Harvard Business Press, Boston, MA; 2009.

[41] Heifetz R. Leadership Without Easy Answers. Harvard University Press, Cambridge, MA: 1998.

[42] Kegan R. The evolving self: Problem and process in human development. Harvard University Press, Cambridge, MA; 1982

[43] Kegan R, Lahey LL. Immunity to change: How to overcome it and unlock the potential in yourself and your organization. Harvard Business Press, Boston, MA 2009

[44] Loehrer S, Feeley D, Berwick D. 10 new rules to accelerate healthcare redesign. Healthcare Executive 2015:66-69. 
[45] Perlo J, Balik B, Swense S, Kabcenell A, Landsman J, Feeley D. IHI Framework for Improving Joy in Work. IHI White Paper. Cambridge, Massachusetts: Intitute for Healthcare Improvement; 2017.

[46] Linzer M, Poplau S, Grossman E, et al. A cluster randomized trial of interventions to improve work conditions and clinical burnout in primary care: Results from the Healthy Work Place 9HWP) Study. J Gen Intern Med. 2015; 30:1105-11.

[47] Pozdnyakova A, Laiteerapong N, Volerman A, et al. Impact of medical scribes on physician and patient satisfaction in primary care. J Gen Intern Med. 2018; 33:1109-1115.

[48] Gidwani R, Nguyen C, Kofoed A, et al. Impact of scribes on physician satisfaction, patient satisfaction, and charting efficiency: a randomized controlled trial. Ann Fam Med. 2017;15:427433.

[49] Shanafelt TD, Dyrbye LN, West CP. Addressing physician burnout: the way forward. JAMA. 2017;317:901-902. 
Table 1: Summary of Leaders' Views

\begin{tabular}{|l|}
\hline \multicolumn{1}{|c|}{ Theme 1: Challenges and Responses of Leadership } \\
\hline Leaders care about maintaining humanistic practice \\
\hline Leaders aware of the challenge presented by the electronic medical record \\
\hline Leaders aware of the negative impact of pressure for financial productivity \\
\hline Leaders aware of the critical importance of good faculty role models \\
\hline Leaders aware of the negative impact of fragmentation of care \\
\hline Theme 2: Policies, Programs, and Practices that Promote Humanistic Care \\
\hline Official policies (e.g., mission and vision statements; recognition and rewards) \\
\hline Educational Programs (e.g., formal curriculum; extracurricular) \\
\hline Informal and external programs (e.g., attending memorial services of patients or colleagues) \\
\hline Bottom Up Approaches (e.g., cultivating a culture of speaking out; nurturing caring attitudes; \\
\hline
\end{tabular}

